\title{
On Mutual Information in Multipartite Quantum States and Equality in Strong Subadditivity of Entropy
}

\author{
F Herbut $\dagger$ \\ Serbian Academy of Sciences and Arts, Knez Mihajlova 35, 11000 Belgrade, Serbia
}

\begin{abstract}
The challenge of equality in the strong subadditivity inequality of entropy is approached via a general additivity of correlation information in terms of nonoverlapping clusters of subsystems in multipartite states (density operators). A family of tripartite states satisfying equality is derived.
\end{abstract}


Two, at first glance unrelated, concepts are investigated in this article: the correlation information connected with nonoverlapping composite subsystems (clusters) of a multipartite quantum system and equality in strong subadditivity (SSA) of entropy. It is shown that the first notion is useful for treating the second one. Needless to state that both concepts are important for quantum information theory.

Let $\rho_{1 \ldots N}$ be a multipartite state (density matrix), $S_{1 \ldots N} \equiv S\left(\rho_{1 \ldots N}\right) \equiv$ $-\operatorname{tr}\left[\rho_{1 \ldots N} \log \left(\rho_{1 \ldots N}\right)\right]$ the corresponding quantum entropy, $\rho_{1} \equiv \operatorname{tr}_{2 \ldots N}\left(\rho_{1 \ldots N}\right), \rho_{2}$ etc. the reductions, and $S_{1}, S_{2}$ etc. the corresponding entropies.

The well known subadditivity of entropy claims that always $S_{12} \leq S_{1}+S_{2}$, and that one has equality if and only if $\rho_{12}=\rho_{1} \otimes \rho_{2}$ [1]. This generalizes to $N$ subsystems.

Lemma 1. For all states $\rho_{1 \ldots N}$ and for $N \geq 2$ the subadditivity

$$
S_{1 \ldots N} \leq \sum_{n=1}^{N} S_{n}
$$

is valid, and one has equality if and only if $\rho_{1 \ldots N}=\left(\Pi^{\otimes}\right)_{n=1}^{N} \rho_{n}, i$. e., if all subsystems are uncorrelated.

Proof. If the lemma is valid for $(N-1)$ subsystems, i. e., if $S_{1 \ldots(N-1)} \leq$ $\sum_{n=1}^{(N-1)} S_{n}$, and one has equality if and only if $\rho_{1 \ldots(N-1)}=\left(\Pi^{\otimes}\right)_{n=1}^{(N-1)} \rho_{n}$, then it is valid also for $N$ subsystems. This is so because the first $(N-1)$ subsystems can be understood as one (composite) subsystem. Then subadditivity for two subsystems implies $S_{1 \ldots N} \leq S_{1 \ldots(N-1)}+S_{N}$. This inequality in conjunction with the preceding one leads to $S_{1 \ldots N} \leq \sum_{n=1}^{N} S_{n}$. One has equality if and only if both inequalities preceding the last one are equalities. These are equivalent to $\rho_{1 \ldots N}=\left[\left(\Pi^{\otimes}\right)_{n=1}^{(N-1)} \rho_{n}\right] \otimes \rho_{N}$. Since the claim is valid for $N=2$, by total induction it is valid for all $N \geq 2$.

The nonnegative quantity

$$
I_{1 \ldots N} \equiv \sum_{n=1}^{N} S_{n}-S_{1 \ldots N}
$$

is called the correlation information (contained) in $\rho_{1 \ldots N}$. For $N=2$, it is called (quantum) mutual information. The correlation information in an $N$-partite state is positive if $\rho_{1 \ldots N}$ is in any way different from the tensor product of all subsystem states.

Let

$$
\Pi: \quad\{1 \ldots N\}=\sum_{k=1}^{K} C_{k}
$$

be an arbitrary partitioning of the set $\{1 \ldots N\}$ into classes, physically, clusters, each consisting of some of the subsystems 1, 2, ., N. (Note that the clusters are nonoverlapping in the subsystems.) Let $\rho_{C_{k}}$ be the reduced density matrix corresponding to the $k-t h$ cluster, obtained by tracing out in $\rho_{1 \ldots N}$ all subsystems except those belonging to the class $C_{k}$. Let, further, $S_{C_{k}}$ be the entropy of this density matrix. Lemma 1 in application to the clusters implies

$$
S_{C_{k}} \leq \sum_{n \in C_{k}} S_{n}
$$


with equality if and only if all subsystems in the cluster are uncorrelated.

Let the correlation information in the cluster $C_{k}$ be

$$
I_{C_{k}} \equiv \sum_{n \in C_{k}} S_{n}-S_{C_{k}}
$$

We call it the within-the-cluster correlation information. (Note that if $C_{k}=\{n\}$, then $I_{C_{k}}=S_{n}-S_{n}=0$.) The correlation information in a composite cluster is zero if and only if all subsystems in the cluster are uncorrelated.

Further, since the clusters can be understood as (composite) subsystems, (1) implies

$$
S_{1 \ldots N} \leq \sum_{k=1}^{K} S_{C_{k}}
$$

and one has equality if and only if all clusters are uncorrelated with each other.

Finally, let

$$
I_{\Pi} \equiv \sum_{k=1}^{K} S_{C_{k}}-S_{1 \ldots N}
$$

be the among-the-clusters correlation information. It is positive if and only if there is any correlation among the clusters. If $K=N$, i. e., if all clusters in the partitioning (3) contain only one subsystem, then (5b) has the special form (2).

Now, we formulate the theorem on cluster additivity of correlation information.

Theorem 1. For every $N$-partite state $\rho_{1 \ldots N}$ the following additivity is valid:

$$
I_{1 \ldots N}=I_{\Pi}+\sum_{k=1}^{K} I_{C_{k}}
$$

In words, the total correlation information is the sum of the among-the-cluster one, and the sum of the within-the-clusters ones summed over all clusters.

Note that (6) is valid for every partitioning $\Pi$.

Proof. Adding and subtracting $\sum_{k=1}^{K} S_{C_{k}}$ on the RHS of (2), one obtains

$$
I_{1 \ldots N}=\left(\sum_{k=1}^{K} S_{C_{k}}-S_{1 \ldots N}\right)+\left(\sum_{k=1}^{K}\left(\sum_{n \in C_{k}} S_{n}-S_{C_{k}}\right)\right)
$$

which, on account of (5b) and (4b), gives (6).

The theorem is a rare statement of great generality that is harder to state than to prove. It implies a useful corollary on successive binary steps.

Corollary 1. One can take $K=2$, then within each cluster repeat this procedure etc. In this way $I_{1 \ldots N}$ is evaluated in terms of binary steps; each step giving a term that is a quantum mutual information.

For $N=3$ both the theorem and the corollary enable one only to make a onestep binary partition; but this can be done in three ways: $\Pi_{1}:\{123\}=\{1\}+\{23\}$, $\Pi_{2}:\{123\}=\{2\}+\{13\}$, and $\Pi_{3}:\{123\}=\{3\}+\{12\}$. We will write $I_{\Pi_{1}}$ as $I_{1,23}$ etc. 
to display the fact that one is dealing with the mutual information between subsystem 1 and the cluster $\{23\}$ etc.

Let us turn to the strong subadditivity (SSA) of entropy for tripartite systems. Its intuitively most appealing form is

$$
I_{12} \leq I_{1,23}
$$

etc. (See [2], Theorem 11.15(2), pp. 522-523. Nielsen and Chuang state only necessity of (7), but prove its equivalence with the standard form of SSA.)

For $N$-partite systems, SSA has the general form which says: Mutual information between two nonoverlapping clusters never increases discarding any number of subsystems from any of the clusters. (It is straightforward to establish equivalence between the general form and (7).)

Strong subadditivity is an inequality. It is interesting to see when it is an equality. There exist sufficient and necessary conditions for SSA equality in the literature, cf [3], [4], 5] (but they are not easily workable).

The simplest concrete example ([3], pp. 4361-4362) is the following:

$$
\rho_{123} \equiv \rho_{12} \otimes \rho_{3}
$$

The cluster additivity then gives utilizing $\Pi_{3}: I_{123}=I_{12,3}+I_{12}=0+I_{12}$. On the other hand, the partition $\Pi_{1}$ gives

$$
I_{123}=I_{1,23}+I_{23}=I_{1,23}+0
$$

Altogether,

$$
I_{12}=I_{1,23}
$$

i. e., we have an equality in SSA (cf $(7))$. One can generalize this.

Corollary 2. If $\rho_{1 \ldots N}=\rho_{1 \ldots M} \otimes \rho_{(M+1) \ldots N}$, then $I_{C_{k}, C_{l}}=I_{C_{k}, C_{l}^{\prime}}$, where $C_{l}$ is a cluster containing all subsystems $(M+1) \ldots N$ and at least one subsystem besides them, $C_{k}$ is a cluster nonoverlapping with $C_{l}$, and $C_{l}^{\prime}$ is obtained from $C_{l}$ by discarding any number of the subsystems $(M+1) \ldots N$.

Proof. Let $\bar{C}_{l}$ be the cluster obtained from $C_{l}$ be removing all subsystems $(M+1) \ldots N$. Then, on account of the fact that clusters can be viewed as (composite) subsystems, (9b) implies

$$
I_{C_{k}, \bar{C}_{l}}=I_{C_{k}, C_{l}} \text {. }
$$

On the other hand, the general form of inequality (7) leads to

$$
I_{C_{k}, \bar{C}_{l}} \leq I_{C_{k}, C_{l}^{\prime}}
$$

and also to

$$
I_{C_{k}, C_{l}^{\prime}} \leq I_{C_{k}, C_{l}}
$$

The two inequalities and the preceding equality finally bear out the claim.

In [3] (p. 4362) it was stated that no other special case has been found. The derivation that follows is a reaction to this challenge. 
Since in case (8) $I_{23}=0$, and the first equality in (9a) is generally valid, one might think that this lack of correlations between subsystems 2 and 3 is the crucial point. This would be a wrong conjecture. We derive now a family of cases of SSA equality (9b) in which $I_{23}>0$.

First we define the notion of a mixture (or a state decomposition) that is biorthogonal.

Definition 1. A state decomposition

$$
\rho_{12}=\sum_{k} w_{k} \rho_{12}^{k}
$$

$\left(\forall k: w_{k}>0, \rho_{12}^{k}>0, \operatorname{tr} \rho_{12}^{k}=1 ; \sum_{k} w_{k}=1\right)$ is biorthogonal if, in terms of the reductions of $\rho_{12}^{k}$,

$$
\forall k \neq k^{\prime}: \quad \rho_{s}^{k} \rho_{s}^{k^{\prime}}=0, \quad s=1,2 .
$$

Now we define a family of states $\rho_{123}$ satisfying the $S S A$ equality (9b).

Theorem 2. Let

$$
\rho_{123}=\sum_{k} w_{k} \rho_{123}^{k}
$$

be a mixture of states such that, tracing out subsystem 3, one obtains a biorthogonal state decomposition. Then, if SSA equality (9b) is valid for each state $\rho_{123}^{k}$ in the mixture (11), then it is valid also for $\rho_{123}$. Further, if there are at least two terms in the decomposition, then $I_{23}>0$ for $\rho_{123}$ unless (11) is a special case of (8).

To prove the theorem, we need four auxiliary lemmas. The first is concerned with implied biorthogonality in (11).

Lemma 2. If one views the tripartite system as a bipartite one, in particular as $\{123\}=\{1\}+\{23\}$, decomposition (11) is biorthogonal.

Proof. Let $\forall k: \rho_{2}^{k} \equiv \operatorname{tr}_{13} \rho_{123}^{k}=\sum_{i} r_{i}^{k}|k i\rangle_{2}\left\langle\left. k i\right|_{2}\right.$ be spectral decompositions in terms of positive eigenvalues. Substitution in (10b) for $s=2$ gives

$$
\forall k \neq k^{\prime}: \quad \sum_{i} \sum_{i^{\prime}} r_{i}^{k} r_{i^{\prime}}^{k^{\prime}}|k i\rangle_{2}\left\langle\left. k i\right|_{2} \mid k^{\prime} i^{\prime}\right\rangle_{2}\left\langle\left. k^{\prime} i^{\prime}\right|_{2}=0\right.
$$

This implies

$$
\forall k \neq k^{\prime}, \quad \forall i, \quad \forall i^{\prime}: \quad\left\langle\left. k i\right|_{2} \mid k^{\prime} i^{\prime}\right\rangle_{2}=0 .
$$

If $R_{2}^{k} \equiv \sum_{i}|k i\rangle_{2}\left\langle\left. k i\right|_{2}\right.$ are the range projectors of $\rho_{2}^{k}$, then one further has

$$
\forall k \neq k^{\prime}: \quad R_{2}^{k} R_{2}^{k^{\prime}}=0 .
$$

One can always write $\rho_{23}^{k}=R_{2}^{k} \rho_{23}^{k}=\rho_{23}^{k} R_{2}^{k}$ (cf relation that is below (12a) in [6]). Hence,

$$
\forall k \neq k^{\prime}: \quad \operatorname{tr}\left[\rho_{23}^{k} \rho_{23}^{k^{\prime}}\right]=\operatorname{tr}\left[R_{2}^{k} \rho_{23}^{k} \rho_{23}^{k^{\prime}} R_{2}^{k^{\prime}}\right]=\operatorname{tr}\left[\left(R_{2}^{k^{\prime}} R_{2}^{k}\right) \rho_{23}^{k} \rho_{23}^{k^{\prime}}\right]=0 .
$$

(Relation (12) has been utilized.)

One can further write

$$
0=\operatorname{tr}\left[\rho_{23}^{k} \rho_{23}^{k^{\prime}}\right]=\operatorname{tr}\left[\left(\rho_{23}^{k}\right)^{1 / 2} \rho_{23}^{k^{\prime}}\left(\rho_{23}^{k}\right)^{1 / 2}\right] .
$$


This implies $\left[\left(\rho_{23}^{k}\right)^{1 / 2} \rho_{23}^{k^{\prime}}\left(\rho_{23}^{k}\right)^{1 / 2}\right]=0$ because the operator is positive (cf Lemma A.1. in [7]). Further, $\rho_{23}^{k^{\prime}}\left(\rho_{23}^{k}\right)^{1 / 2}=0$ (cf Lemma A.2. in [7]). Multiplying this from the right by $\left(\rho_{23}^{k}\right)^{1 / 2}$, one finally obtains

$$
\forall k \neq k^{\prime}: \quad \rho_{23}^{k} \rho_{23}^{k^{\prime}}=0 .
$$

This, in conjunction with with (10b) for $s=1$, completes the proof.

Thew next lemma concerns the mixing property of mutual information for biorthogonal mixtures.

Lemma 3. Mutual information of a biorthogonal mixture with weights $w_{k}$ equals the sum of the Shannon entropy $H\left(w_{k}\right) \equiv-\sum_{k} w_{k} l o g w_{k}$ and the average mutual informations of the states that are mixed.

This lemma was proved in [8] (see Lemma 9 there).

Next we state and prove the generalized mixing property of entropy of any mixture, which is known, but perhaps not well known.

Lemma 4. Let

$$
\rho=\sum_{k} w_{k} \rho^{k}
$$

be any state decomposition. Then the following entropy decomposition is valid:

$$
S(\rho)=\sum_{k}\left[w_{k} S\left(\rho^{k} \| \rho\right)\right]+\sum_{k}\left[w_{k} S\left(\rho^{k}\right)\right]
$$

where $S(\rho \| \sigma) \equiv \operatorname{tr}(\rho \log \rho)-\operatorname{tr}(\rho \log \sigma)]$ is the relative entropy of the corresponding states (if the support of $\sigma$ contains that of $\rho$ ).

Proof. First we must prove that

$$
\forall k: \quad \operatorname{supp}\left(\rho^{k}\right) \subseteq \operatorname{supp}(\rho) .
$$

(By "support" one means the subspace that is the topological closure of the range.) In view of the fact that the support of a density matrix $\sigma$ is spanned by any set of pure states into which $\sigma$ can be decomposed (cf Appendix(ii) in 9]), one should decompose each $\rho^{k}$ into pure states and substitute in (14a). Then (15) obviously follows.

By substituting (14a) in part of (14b) (though not everywhere), one obtains:

$$
\begin{gathered}
S(\rho)-\sum_{k}\left[w_{k} S\left(\rho^{k}\right)\right]=-\operatorname{tr}\left\{\sum_{k}\left[w_{k} \rho^{k} \log (\rho)\right]\right\}+\sum_{k}\left\{w_{k} \operatorname{tr}\left[\rho^{k} \log \left(\rho^{k}\right)\right]\right\}= \\
\sum_{k}\left\{w_{k}\left[-\operatorname{tr}\left(\rho^{k} \log \rho\right)+\operatorname{tr}\left(\rho^{k} \log \rho^{k}\right)\right]\right\}=\sum_{k}\left[w_{k} S\left(\rho^{k} \| \rho\right)\right] .
\end{gathered}
$$

Remark 1. In the special case when (14a) is an orthogonal state decomposition, i.e., when $\forall k \neq k^{\prime}: \rho^{k} \rho^{k^{\prime}}=0$, then (14b) takes on the well known special form

$$
S(\rho)=H\left(w_{k}\right)+\sum_{k}\left[w_{k} S\left(\rho^{k}\right)\right]
$$

where $H\left(w_{k}\right)$ is the Shannon entropy of the probability distribution $\left\{w_{k}: \forall k\right\}$. One refers to (16) as the mixing property of entropy ([1]). 
Next we define the concept of a monoorthogonal mixture.

Definition 2. A state decomposition

$$
\rho_{23}=\sum_{k} w_{k} \rho_{23}^{k}
$$

is called monoorthogonal in subsystem 2 if one has

$$
\forall k \neq k^{\prime}: \quad \rho_{2}^{k} \rho_{2}^{k^{\prime}}=0
$$

in terms of the corresponding reductions.

Now we state a lemma on the mixing property of mutual information for monoorthogonal mixtures.

Lemma 5. If one has a mixture (17a) monoorthogonal in subsystem 2, the mutual information $I_{23}$ of the decomposed state can be written as follows:

$$
I_{23}=\sum_{k} w_{k} S\left(\rho_{3}^{k} \| \rho_{3}\right)+\sum_{k} w_{k} I_{23}^{k}
$$

Proof. The argument is straightforward in view of (17a) and (17b) with the help of (16):

$$
\begin{gathered}
I_{23} \equiv S_{2}+S_{3}-S_{23}=\left\{H\left(w_{k}\right)+\sum_{k}\left[w_{k} S\left(\rho_{2}^{k}\right)\right]\right\}+ \\
\left\{\sum_{k}\left[w_{k} S\left(\rho_{3}^{k}|| \rho_{3}\right)\right]+\sum_{k}\left[w_{k} S\left(\rho_{3}^{k}\right)\right]\right\}-\left\{H\left(w_{k}\right)+\sum_{k}\left[w_{k} S\left(\rho_{23}^{k}\right)\right]\right\} .
\end{gathered}
$$

After cancellation and substitution of $I_{23}^{k}$, the claimed relation (18) ensues.

Proof of the theorem: On account of the implied biorthogonality (Lemma 2), we can apply the mixing property of Lemma 3 to decomposition (11). Hence

$$
I_{1,23}=H\left(w_{k}\right)+\sum_{k} w_{k} I_{1,23}^{k}
$$

Lemma 3 can also be applied to decomposition (10a) when it is obtained by tracing out subsystem 3 in (11):

$$
I_{12}=H\left(w_{k}\right)+\sum_{k} w_{k} I_{12}^{k}
$$

Since by assumption $\forall k: I_{12}^{k}=I_{1,23}^{k}$, the last two relations bear out the first claim of the theorem.

Finally, a glance at (18) reveals that for $K \geq 2$, one can have $I_{23}=0$ if and only if $\forall k: \quad \rho_{123}^{k}=\rho_{12}^{k} \otimes \rho_{3}$. This would reduce it to case (8).

Remark 2. Utilizing in Theorem 2 (8) for each state $\rho_{123}^{k}$, but possibly with distinct factor states for different values of $k$, one obtains various concrete states $\rho_{123}$ satisfying (9b).

Let us return to inequality (7). There are 6 distinct inequalities of this type (obtained from (7) by permutations). Each of them defines a nonnegative excess in 
mutual information, e. g., $\left(I_{1,23}-I_{12}\right)$. Two by two of the 6 excesses are equal. For example

$$
I_{1,23}-I_{12}=I_{12,3}-I_{23}
$$

Equality (19) is obvious if rewritten as

$$
I_{1,23}+I_{23}=I_{12,3}+I_{12}
$$

when it is an instance of the cluster additivity of correlation information in the tripartite system. Zero excess is the same thing as equality in SSA of entropy. Thus, (9b) gives rise to

$$
I_{23}=I_{12,3}
$$

and vice versa.

When (9b) is valid, relation (20) is a new equality in SSA. In case (8), (20) is trivial, because subsystem 3 has zero mutual information both with subsystems 2 and 12, and then also the excess is zero. In the case treated in Theorem 2, (20) is a, perhaps even surprising, new result.

Finally, let us see if in the case defined in Theorem 2 one can, in the spirit of Corollary 2, replace subsystem 3 by a composite system, a cluster, and discard not the whole cluster, but only part of it, and still have no decrease in mutual information with another (nonoverlapping) cluster. An affirmative answer follows from realizing that in the proof of Corollary 2 all that was used was the possibility of discarding the whole cluster (equality $I_{C_{k}, \bar{C}_{l}}=I_{C_{k}, C_{l}}$ ), and two SSA inequalities. All three are valid also in the present case.

In conclusion, one may say that the general cluster additivity of correlation information (Theorem 1), used through successive binary partitionings (Corollary 1), gave a useful view of correlations. It made possible generating new equalities (cf (20)), as well as generalization to clusters (cf Remark 2 and the preceding passage). The main result is the family of states $\rho_{123}$ satisfying equality in SSA of entropy (Theorem 2).

[1] A. Wehrl, Rev. Mod. Phys. 50, 221 (1978).

[2] M. A. Nielsen and I. L. Chuang, Quantum Computation and Quantum Information (Cambridge Univ. Press, Cambridge UK, 2000).

[3] M. B. Ruskai, J. Math. Phys. 43, 4358 (2002).

[4] M. Ohya and D. Petz, Quantum Entropy and Its Use, (Springer-Verlag, New York, 1993).

[5] D. Petz, Commun. Math. Phys. 105, 123 (1986).

[6] F. Herbut and M. Damnjanović, J. Phys. A: Math. Gen. 33, 6023 (2000).

[7] F. Herbut, Found. Phys. Lett. 9, 437 (1996).

[8] F. Herbut, J. Phys. A: Math. Gen. 36, 8479 (2003).

[9] F. Herbut, "Mixing Property of Quantum Relative Entropy", quant-ph/0309211 